\title{
“TODOS LOS INSTRUMENTOS ESTÁN EN BUEN ESTADO". DISPUTAS EN TORNO AL FUNCIONAMIENTO DE LOS TELESCOPIOS DEL OBSERVATORIO ASTRONÓMICO NACIONAL DE CHILE EN EL SIGLO XIX ${ }^{1}$
}

\author{
Carlos Sanhueza-Cerda \\ Universidad de Chile \\ Email: carlos.sanhueza@uchile.cl \\ ORCID iD: https://orcid.org/0000-0002-6640-3473 \\ Lorena B. Valderrama \\ Universidad Alberto Hurtado \\ Email: Ivalderrama@uahurtado.cl \\ ORCID iD: https://orcid.org/0000-0002-3794-692X \\ Stefan Meier \\ Universidad de Chile \\ Email: smeier@ug.uchile.cl \\ ORCID iD: https://orcid.org/0000-0002-7614-3425 \\ José Soto \\ Universidad de Chile \\ Email: jose.sotovejar@gmail.com \\ ORCID iD: https://orcid.org/0000-0002-9957-5116
}

Recibido: 14 noviembre 2018; Aceptado: 23 julio 2019

Cómo citar este artículo/Citation: Sanhueza-Cerda, Carlos; Valderrama, Lorena B.; Meier, Stefan; Soto, José (2020), "'Todos los instrumentos están en buen estado'. Disputas en torno al funcionamiento de los telescopios del Observatorio Astronómico Nacional de Chile en el siglo XIX", Asclepio, 72(1): p300. https://doi.org/10.3989/asclepio.2020.09.

RESUMEN: El artículo analiza una controversia pública sobre el estado de los principales instrumentos del Observatorio Astronómico Nacional de Chile a fines del siglo XIX, las labores de reparación y mantención de estos y la producción científica de la institución. En la controversia participaron una amplia gama de actores, entre los que se encuentran miembros del Congreso Nacional, ministros de Estado, astrónomos, mecánicos y arquitectos. Este caso permite examinar la estrecha vinculación entre la esfera política y la tecnocientífica, evidenciándose en la administración, financiamiento, evaluación y fiscalización del quehacer del observatorio astronómico. Acá podemos observar cómo el cuestionamiento sobre la calidad científica, como el estado de los instrumentos de la institución, enfrentó no solo los juicios, sino la autoridad de quienes los emitían. Por otro lado, esta controversia permite observar cómo la necesidad de crear tecnologías asociadas y adaptar los instrumentos astronómicos dio un protagonismo a mecánicos, ingenieros y arquitectos, actores muchas veces invisibles en comparación con los astrónomos.

PALABRAS CLAVE: Observatorio Astronómico Nacional; Controversias; Reparación; Mantención; Telescopios.

"ALL INSTRUMENTS ARE IN GOOD CONDITION". DISPUTES SURROUNDING THE OPERATION OF THE TELESCOPES AT THE NATIONAL ASTRONOMICAL OBSERVATORY OF CHILE IN THE 19TH CENTURY

ABSTRACT: This paper analyses a public controversy about the state of the main instruments at the National Astronomical Observatory of Chile at the end of the 19th century, the repair and the maintenance work of these tools and the scientific production of this institution. The controversy involved a wide range of actors, including members of the National Congress, ministers of State, astronomers, mechanics and architects. This case examines the close link between the political and the techno-scientific spheres, evidenced in the administration, financing, evaluation and oversight of the work of the observatory. We can observe how the questioning of scientific quality, such as the state of the instruments of the institution, faced not only the judgments, but also the authority of those who issued. Also, this controversy allows us to observe how the need to create associated technologies and adapt astronomical instruments gave prominence to mechanics, engineers and architects, actors often invisible in comparison to astronomers.

KEY WORDS: National Astronomical Observatory; Controversies; Repair; Maintenance; Telescopes.

Copyright: ( 2020 CSIC. Este es un artículo de acceso abierto distribuido bajo los términos de la licencia de uso y distribución Creative Commons Reconocimiento 4.0 Internacional (CC BY 4.0). 


\section{INTRODUCCIÓN}

El presente artículo examina una controversia pública en torno al estado y uso de los telescopios del Observatorio Astronómico Nacional de Chile (OAN) hacia fines del siglo XIX. Los cuestionamientos se centraron en la responsabilidad de su director, José Ignacio Vergara, en el resguardo y uso adecuado de los instrumentos. Estas denuncias surgieron en una publicación sobre la situación de las instituciones científicas en Chile, lo que instaló una discusión en el Congreso Nacional en la que intervinieron algunos diputados, pero también funcionarios del OAN, quienes disputaban la legitimidad para evaluar el buen funcionamiento de los telescopios.

Este caso permite analizar la estrecha vinculación entre la esfera política y la tecnocientífica, tanto en la administración, financiamiento, evaluación y fiscalización del quehacer del observatorio astronómico. Acá podemos observar cómo el cuestionamiento sobre la calidad científica y el estado de los instrumentos de la institución enfrentó no solo los juicios, sino la autoridad de quienes los emitían. ¿Quién tiene la palabra autorizada: el político que debe velar por el buen uso de los fondos públicos, el técnico encargado de la mantención de los equipos o el astrónomo que debe hacer las mediciones y observaciones? Por otro lado, el estudio de esta controversia permite acercarse al fenómeno de los states of disrepair (Schaffer, $2011,708)$. Schaffer sostiene que en la reparación de instrumentos tiende a verse oscurecido el papel de los técnicos o artesanos en la medida en que no dejan registro de estas acciones. Este estudio de caso espera visibilizar a estos actores.

La primera parte del artículo da cuenta de cómo el estudio de las controversias científicas ha sido abordado por la literatura internacional. En la segunda parte se analiza la tensión entre las funciones políticas y científicas que ejercía el director del OAN. La tercera parte examina la repercusión que tenía el estado de los instrumentos en la calidad de la producción científica en el caso de estudio. En la cuarta y última parte se analiza la importancia que desempeñaron las tecnologías asociadas a dichos instrumentos.

\section{CONTROVERSIAS, POLÉMICAS Y DISPUTAS}

Desde diversas disciplinas de las ciencias sociales y humanidades se ha propuesto estudiar detenidamen- te las controversias y polémicas tecnocientíficas por ser una parte esencial de las actividades y prácticas científicas y tecnológicas, no constituyendo estas excepciones o anomalías ( Engelhardt y Caplan, 1987; Latour, 1991; Dascal, 1998; Machamer, Darden y Craver, 2000). Sin embargo, pese a su importancia para comprender el quehacer científico, su tipología resulta difusa, dado que su definición es laxa. Para algunos autores una controversia científica es una disputa cuyo objeto está claramente definido: sucede entre dos o más actores que saben que están envueltos en la controversia; se encuentra claramente delimitada en el tiempo; tiene un carácter epistémico y se soluciona con el reconocimiento de un error o mala interpretación, o bien, se disuelve mediante algún procedimiento arbitrario, como por ejemplo recurrir a alguna autoridad científica (Freudenthal, 1998; Dascal, 1998; Sismondo, 2010) ${ }^{2}$. Para otros autores la controversia difícilmente se logra delimitar en el espacio, tiempo y actores exactos que conforman el debate, ni sus resoluciones son tan claras (Latour, 1991).

Independiente de su tipología, como herramienta analítica ha demostrado su utilidad y para ello el Programa Fuerte, y los estudios STS en general, proponen que metodológicamente un análisis de controversias, debates y disputas tecnocientíficas debe ser necesariamente de tipo simétrico (Sismondo, 2010; Dascal, 1998). Es decir, se deben analizar los mismos elementos para todas las partes en disputa. Otros ponen énfasis en que se debe evitar

debates esencialistas sobre quién posee o deja de poseer las supuestas 'verdades' de la ciencia, y analizar a cada uno de los protagonistas desde la perspectiva de su formación, retribución, prácticas, experimentos, publicaciones y otras estrategias de socialización (NietoGalan, 2011, p. 128).

Si bien se tiende a suponer que la mayoría de las disputas tiene dos partes en conflicto claramente definidas, esto no siempre es así (Jasanoff, 1996). Los participantes toman posiciones de acuerdo a sus intereses personales, profesionales, económicos o políticos, entre otros (MacKenzie, 1978). Además, estas posiciones pueden ir mutando en el tiempo. Analizar los argumentos debatidos, los espacios donde éstos se discutieron, así como también los instrumentos, técnicas, métodos o estrategias discursivas utilizadas, se vuelve relevante para dilucidar los intereses políticos, económicos, disciplinares y personales de aquellos involucrados en los debates (Shapin y Schaffer, 
1985; Cambrosio, Keating y MacKenzie, 1990; Beder, 1991; Livingstone, 2007; Sismondo, 2010). En este sentido, las herramientas que despliegan los actores para convencer a otros juegan un papel importante dentro de las controversias.

Muchos investigadores han estudiado las controversias sociotécnicas dentro de las comunidades disciplinares, sin embargo, resulta cada vez más interesante seguir la huella de estas disputas dentro del ámbito público. En efecto, el traslado de un controversia a otras esferas permite estudiar ciertos intereses sociales, políticos y económicos que tienen los involucrados, como por ejemplo: que se les reconozca como autoridades en determinadas materias; su interés en obtener financiamiento para sus investigaciones o la búsqueda de una legitimación social (Shapin y Barnes, 1977; Beder, 1991). Más aún, las propuestas de las corrientes constructivistas de la tecnología convocan a los investigadores a no separar lo político o social de lo técnico (Bijker, Hughes y Pinch, 1987). Tanto lo técnico como lo político se constituirían como esferas interrelacionadas y coconstitutivas (Edwards y Hecht, 2010).

Al igual que en la disputa sobre las imperfecciones de los grandes telescopios entre astrónomos "amateurs" e institucionales en Gran Bretaña durante las últimas décadas del siglo XIX (Lankford, 1981), la disputa en torno al estado de los instrumentos del Observatorio Astronómico Nacional de Chile a finales del siglo XIX, permite observar dinámicas de la práctica científica dentro de la institución en cuanto al uso, mantención y reparación de los artefactos usados para la observación, como también el papel que desempeñaron las instituciones científicas para la sociedad, la fiscalización del quehacer científico y los discursos sociales en torno a la generación del conocimiento astronómico a finales del siglo XIX.

El desperfecto y desajuste de los instrumentos junto a su deseada perfección y ajuste, fue la clave en esta controversia. En efecto, estas acciones son relevantes pues otorgan estabilidad, permitiendo la habilitación de prácticas y trabajos cotidianos dentro de las instituciones científicas. Aunque los instrumentos muchas veces pasen desapercibidos dentro de las rutinas científicas diarias (Graham y Thrift, 2007), al momento de su rompimiento o desperfecto, desarticulan el trabajo científico y necesitan ser reparados para que este vuelva a su flujo normal (Jackson, 2014; Potthast, 2007) $)^{3}$. De esta manera, la mantención y reparación funciona como una bisagra entre el inevi- table fenómeno del rompimiento y desperfecto y el funcionamiento expedito de la práctica científica (Jackson, 2014).

En estos momentos de desperfecto y desajuste los técnicos o mecánicos encargados de reparar y mantener se hacen visibles y protagonistas. No obstante, estos actores se han mantenido en el anonimato por largo tiempo. Este manto de invisibilidad, como lo ha planteado Shapin (1989), se explica tanto por la situación misma de los técnicos como por la concepción del trabajo científico y la producción de conocimiento. Separado el trabajo intelectual del manual, la historia occidental ha subvalorado este último, quitándole la posibilidad de ser reconocido como generador válido de conocimiento (Morus, 2016). De esta forma, no ha sido extraño que, tanto en el discurso científico como en la historia de la ciencia, los técnicos solo aparezcan en pocas ocasiones. Pese a esta carga histórica, existen algunas situaciones en las que los técnicos se hacen visibles, tales como accidentes en los laboratorios, experimentos fallidos o disputas entre técnicos y científicos (Shapin, 1989; Morus, 2016). En este sentido, tanto las controversias como las acciones de reparación y mantención son una puerta para conocer y evaluar la labor de los técnicos.

\section{LA INCOMPATIBILIDAD DE LO CIENTÍFICO Y LO POLÍTICO}

En 1885 varios diputados habían solicitado al ministro de Justicia, Culto e Instrucción Pública datos sobre los trabajos científicos del OAN e incentivos de productividad de sus trabajadores. Sin embargo, la petición no recibió respuesta durante ese año y tampoco se discutió extensamente en el Congreso. El 3 de febrero de 1886, en cambio, el diputado conservador Manuel Balbontín (1845-1918) solicitó al ministro de Instrucción Pública que explicara el estado en el que se encontraba el OAN "y cuanto se ha gastado en instrumentos y refacciones en el último año" ${ }^{\prime 5}$. A su juicio, el observatorio estaba en condiciones deplorables, opacando el liderazgo internacional que tuvo en administraciones previas. Es posible afirmar que esta queja pudo haber nacido no de un interés genuino por el estado y quehacer científico nacional, si no por razones propiamente político partidistas. El año 1886 fue de elecciones presidenciales en Chile, en un contexto social de constantes sospechas por fraude electoral (Portales, 2004). Hasta ese 
entonces, el gobierno era liderado por el abogado Domingo Santa María (1824-1889), miembro del Partido Liberal ${ }^{6}$. El OAN, a su vez, estaba dirigido por el ingeniero geógrafo José Ignacio Vergara (1837-1889) ${ }^{7}$, miembro también del Partido Liberal, quien era además desde 1885 ministro del Interior y, como tal, le correspondía hacerse cargo del proceso electoral ${ }^{8}$.

Pese a las razones personales o políticas que pudieron haber iniciado esta controversia pública sobre el estado de los instrumentos del OAN, el cariz que fue tomando a lo largo de los meses y sus consecuencias para los astrónomos de la institución permiten analizar aspectos claves sobre las experticias en torno a los instrumentos. ¿Quién era la persona indicada para discernir la utilidad y calidad de un instrumento científico: quien realizaba las observaciones, quien se encargaba de su manutención o quien se encargaba de repararlos? Más aún, ¿estaba el político capacitado para juzgar el estado general de una institución científica y la calidad de su productividad?

Esta controversia registrada en el Parlamento, además es significativa porque refleja una profunda imbricación entre lo político y lo tecnocientífico. La figura de Vergara es el más claro ejemplo: un ministro de Estado como director de un observatorio astronómico estatal, en un periodo donde esta doble función científica-política no era una excepcionalidad y estaba amparada por la ley. Asimismo, esta controversia iniciada en el Congreso generó divergencias en el equipo interno del observatorio, enfrentó experticias y concluyó con el despido de su primer astrónomo, el alemán doctor en astronomía Adolf Marcuse ${ }^{9}$.

Para comprender la temporalidad de la controversia y sus argumentos, es importante señalar que en un primer momento el cuestionamiento dentro del Parlamento se debió al estado físico de los instrumentos. El Ministerio de Instrucción Pública (del cual dependía institucionalmente el observatorio), argumentó que esto se debía a una falta sostenida de presupuesto ${ }^{10}$. Sin embargo, para los diputados el problema parecía ser aún más profundo. Según ellos, las labores científicas eran incompatibles con las labores políticas. En particular, el diputado militante del partido radical y férreo opositor al gobierno de Santa María, Guillermo Puelma Tupper (1851-1895) ${ }^{11}$, acusó a José Ignacio Vergara de "abandonar" la institución científica para dedicarse a labores políticas de gobierno ${ }^{12}$. Vergara desestimó esta acusación y aclaró que su cargo como director era ad-honorem, pero coincidía con sus acusadores en que las labores administrativas eran in- compatibles con las propiamente científicas. Esta era la razón por la cual, según él, no se dedicaba de forma personal a las observaciones astronómicas. Según el ministro-director, para ejecutar estas tareas estaban contratados los otros astrónomos del observatorio: desde 1884 lo estaba el segundo astrónomo, Wilhelm Wickmann y, próximo a llegar a Chile en febrero de 1886, también lo estaría el primer astrónomo Adolf Marcuse (1860-1930) ${ }^{13}$.

En el transcurso de los meses, y a medida que avanzaba la discusión en la Cámara, fue tomando más fuerza la crítica respecto de la gestión de los recursos y los diputados comenzaron a responsabilizar a la dirección del OAN por no haber gastado dinero en instrumentos de observación, ni en sus refacciones. La falta de instrumentos adecuados se consideró la principal razón por lo cual la producción científica de la institución era menor a la de otros observatorios extranjeros $^{14}$.

Para agosto de 1886, el juicio de los parlamentarios era lapidario. En palabras del diputado Puelma Tupper: "Pocas veces han podido ser defraudadas más completamente las esperanzas que el país tenía derecho a fundar en un plantel científico creado bajo tan buenos auspicios"15. Ante los cuestionamientos de los parlamentarios de la Cámara de Diputados, hubo una réplica en la prensa por parte del segundo astrónomo de la institución, Wilhelm Wickmann. Como trabajador, Wickmann no podía contestar directamente a las intervenciones de los políticos en el parlamento, por ello la prensa se constituyó como el espacio idóneo para sus réplicas. En estas se anunciaba el problema de la autoridad y competencia que tenían algunos actores (como los políticos) para juzgar el quehacer científico de la institución. Para el segundo astrónomo del observatorio, por ejemplo, sujetos que no tenían conocimientos astronómicos no podían ni debían pronunciarse respecto a los trabajos científicos producidos por la institución:

Es mui estraño para mí que trabajos astronómicos, sobre todo, siendo ellos orijinales, sean pedidos para presentarlos y exijir un juicio sobre ellos a un público que no puede entender gran cosa de estas especialidades, en las que, ni siquiera tienen para qué ocuparse injenieros y marinos; pero mas estraño es todavia el juicio que el señor Puelma enunció sobre estos trabajos en la sesion del 12 de agosto. El que quiera formarse un juicio sobre el valor de trabajos astronómicos, no solo debe ser un astrónomo, sino que tambien debe conocer perfectamente la calidad y la construccion de los respectivos instrumentos ${ }^{16}$. 
Paralelamente a las discusiones dentro del parlamento, el diputado por el Partido Liberal, Jacinto Chacón (1820-1893) ${ }^{17}$, realizó una inspección autónoma para informar sobre la situación de las instituciones científicas chilenas financiadas por el Estado. Chacón elaboró un completo informe sobre la llamada "Quinta Normal de Agricultura", espacio dedicado al cultivo de las ciencias y el desarrollo tecnológico en Chile, que a fines del siglo XIX contaba en su seno con un Museo de Historia Natural, un Jardín Botánico, un Instituto Agrícola y el Observatorio Astronómico Nacional. Para referirse a este último contó con ayuda del recién llegado primer astrónomo de la institución, Adolf Marcuse. Según Chacón, el objetivo de su informe era

manifestar la importancia (...) de todos estos establecimientos para que una vez, conocidos y apreciados en todo su valor, sean ellos vigilados por la opinión, el Congreso y el Gobierno, a fin de que se les dote de los elementos necesarios ${ }^{18}$.

El texto de Chacón se publicitó como un ejemplo de que la labor de estas instituciones no era percibido como un aspecto aislado de la sociedad, sino como un asunto esencialmente público, resaltando la importancia de la fiscalización de estos establecimientos por parte del aparato político. De igual forma, se dieron argumentos públicos de la experticia y pertinencia del autor para juzgar el estado y condiciones de las instituciones científicas analizadas en el libro:

Ocurre a primera vista la duda de que un literato i comentador de nuestras leyes codificadas no parece propio que se dedique a escribir sobre asuntos que, al parecer, están fuera de la órbita de sus conocimientos i de sus cuotidianas tareas del foro i el bufete de abogado. Sin embargo, el que así piense o discurra padecerá un gravísimo error. El conocido autor de La Quinta Normal pertenece al escaso número de los que entre nosotros gozan del envidiable privilejio de ser doctos maestros no solo en lo que dice inmediata relacion con sus trabajos profesionales sino tambien en muchas otras esferas de conocimientos. El señor Chacon que desde los comienzos de su carrera literaria se distinguió por su levantada inspiracion de poeta orijinal i fecundo, vióse mas tarde, cuando el peso de la edad aquilata el criterio, llevado a otra suerte de estudios, i entónces [sic] se hizo jurisconsulto de los mas distinguidos de nuestro pais, abogado de justa nombradía, poeta de estro vigoroso de tarde en tarde, i hombre de ciencia que así conoce las fórmulas del áljebra i la trigonometría, los progresos astronómicos i de la jeolojía como los encantos que proporcionan las ciencias físico-químicas. En el autor del libro que hoi nos proponemos analizar a la lijera se ven, pues, en íntimo i provechoso maridaje el arte i la ciencia ${ }^{19}$.
La incompatibilidad de lo científico y lo político, denunciada por los parlamentarios, no solo era respecto a la delimitación de ambas esferas, sino puesto que a la larga repercutía en una mala gestión y, por ende, en una baja productividad de la institución. Sin embargo, Vergara había tratado de compatibilizar su cargo como astrónomo y político: había sido nombrado director interino del OAN en 1865 y fue diputado por Talca entre 1867-1870, al mismo tiempo que publicaba en 1866 los trabajos "Futuro Eclipse" y "Observación Meridiana" ${ }^{20}$. Al asumir más adelante como director en propiedad del OAN (1874), fue nombrado intendente de Talca, cargo que mantuvo hasta 1881. Luego desempeño cargos de mayor envergadura y responsabilidad en el gobierno: fue ministro de Justicia, Culto e Instrucción Pública en 1883, ministro subrogante de Guerra y Marina en 1884 y desde 1885 se desempeñó también como ministro del Interior. Durante los últimos diez años como director del OAN, su producción científica mermó cuantitativamente: entre 1877 y 1883 sólo había producido 2 trabajos, en comparación con los 8 que produjo entre 1866 y 1873.

Ahora bien, más allá del tema de la productividad de la institución y su liderazgo (o no liderazgo) internacional, el tema del estado de los instrumentos y la exactitud de los datos que éstos podían entregar fue el que más repercutió tanto interna como externamente al observatorio, como se verá en el apartado siguiente.

\section{INSTRUMENTOS PERFECTOS, DATOS EXACTOS}

En el capítulo dedicado al OAN del informe de Chacón, éste realizó en todo momento mención al estado de los instrumentos: era indispensable mantener los instrumentos "perfeccionados" para que pudieran dar "al observador datos exactos para sus deducciones astronómicas" 21 . Con esto se refería a que la astronomía "necesita de instrumentos perfectos y bien montados" pues no es "ciencia de la inducción" (...) al contrario, [es] ciencia exacta y de observación que necesita datos para fundar sus teorías y sus cálculos matemáticos"22. Incluso, dedicó un capítulo especial para abordar el estado, reparación y adquisición de instrumentos astronómicos, haciendo un relato en el que se detallaban objetos utilizados desde la fundación del OAN hasta 1886. 
Por su parte, los parlamentarios también hicieron hincapié en la exactitud de la producción científica del observatorio. El 12 de agosto, el diputado Puelma Tupper pidió al ministro la recopilación de los manuscritos de observaciones realizadas en el OAN, además de la labor realizada hasta ese momento por el primer astrónomo Adolf Marcuse, para formarse una opinión. Según él: "Los trabajos del Observatorio que se han publicado, de lo único que dejan constancia es del descuido con que han sido hechos y de las inexactitudes de que adolecen"23. En específico, se cuestionó el estado de cinco instrumentos del observatorio: dos ecuatoriales, dos círculos meridianos y un busca-cometas.

Este cuestionamiento político sobre el estado de los instrumentos implicó discusiones técnicas entre los miembros de la institución. De esta forma, los trabajadores del observatorio presentaron informes y contra-informes al Ministerio de Instrucción Pública, entregando sus versiones sobre el estado de los instrumentos, la calidad de la producción científica y sus opiniones sobre quiénes eran los responsables de ambos asuntos. Para algunos trabajadores de la institución, como el primer astrónomo Marcuse, la cantidad y precisión de los trabajos astronómicos realizados guardaban directa relación con el estado de los instrumentos. En su caso particular, reclamaba que no podía realizar observaciones astronómicas de forma regular y exacta, aspecto que también había dificultado el trabajo del segundo astrónomo Wickmann:

(...) los pocos trabajos que he ejecutado no tienen importancia científica, cuando era imposible, por el mal estado de los instrumentos, hacer observaciones que sirviesen para algo en la astronomía y (...) Wickmann expresa categóricamente que no ha podido, por esa misma causa, trabajar durante casi dos años en el Observatorio ${ }^{24}$.

El informe de Marcuse apuntó a la mala administración de Vergara por la subutilización de los instrumentos y descuido en los trabajos de reparación y mantención que debía hacer el mecánico a cargo, Luis Grosch $^{25}$. El director del observatorio, a su vez, acusó de la baja productividad científica de la institución al primer astrónomo Marcuse. Los trabajadores, incluyendo a Wickmann y Grosch, decidieron apoyar al director-ministro en su acusación contra Marcuse.

Las partes enfrentadas no coincidían en su diagnóstico respecto del problema, ni tampoco sobre los responsables: había diferentes versiones con respecto al estado de los instrumentos, su capacidad y calidad de uso. Vergara, Wickmann y Grosch (el director, el segundo astrónomo y el mecánico) se enfrentaron al primer astrónomo (Marcuse) defendiendo a la institución, su productividad y el buen estado de los instrumentos. El primer astrónomo en cambio se mantuvo fiel hasta el final en su denuncia, acusando la existencia de instrumentos en mal estado que generaban datos inexactos. Por ejemplo, para Marcuse el circulo meridiano estaba dañado. Sin embargo, Grosch y Wickmann afirmaban públicamente que este instrumento funcionaba perfectamente y la prueba de ello era que se utilizaba para dar la hora oficial a la Estación Central de Ferrocarriles y al cerro de Santa Lucía en Santiago.

La disputa interna del OAN que se había transferido al Parlamento chileno tenía una data mayor. Una carta escrita en 1885 por Wickmann al director del Observatorio Nacional de Brasil, Luiz Cruls, prueba la controversia respecto de la reparación de instrumentos entre el director, el segundo astrónomo y el mecánico. En la misiva Wickmann denunciaba que el segundo ecuatorial era un instrumento viejo e inservible, debido a que su micrómetro filar estaba inutilizable y su círculo torcido, sumado a que el micrómetro anular estaba en las mismas condiciones. Wickmann también acusaba en 1885 que el Gran Ecuatorial estaba prácticamente sin uso y dañado producto de su exposición al aire libre: con desechos de aves y puntas de fierro enmohecidas ${ }^{26}$.

El propio Wickmann, en diciembre de 1885 , le escribió a Marcuse contándole del mal estado de los instrumentos del OAN, dos meses antes de que este último llegara a Chile. En esta misiva, Wickmann le contaba a su connacional que el mecánico Luis Grosch (que llevaba 32 años trabajando en la institución) había dañado la inclinación de un círculo meridiano al realizar una limpieza de los pivotes, haciendo que perdieran su forma cilíndrica. Dado que el instrumento no permitía una observación precisa, el segundo astrónomo había solicitado al director enviar el círculo meridiano de vuelta a la fábrica original en Europa para su reparación. Sin embargo, esta solicitud había sido rechazada por Vergara, quien habría afirmado que "no se pueden hacer las cosas con tanta exactitud, en otros Observatorios las cosas tampoco se hacen con tanta prolijidad"27.

Cuando Wickmann encaró al director de la institución, amenazando con no realizar más observaciones con el instrumento, el director envió al me- 
cánico para que examinara el artefacto. Esta acción generó una disputa en la que se enfrentaron los saberes del mecánico y del científico: Wickmann desacreditó la experticia del mecánico en la evaluación del estado del instrumento, explicando que le "era del todo indiferente lo que ese hombre dijese o examinase, que no era costumbre en otros países preguntar cuestiones astronómicas a un zapatero, sastre o mecánico, que lo mismo me importaba lo que dijera el portero" ${ }^{28}$. Por otro lado, el mecánico, quien realizó una serie de nivelaciones en el instrumento, le explicó al director que "el instrumento se hallaba en excelente estado, todo lo que Wickmann dice son tonterías, él no entiende nada de astronomía, las observaciones con ese instrumento se hacen con perfecta exactitud...!"29. Esta declaración fue sostenida por el mecánico también al año siguiente, dejando en claro que "El círculo meridiano del ala derecha, o como otros lo llaman, el viejo círculo, está en buen estado de arreglo y en actitud de ser usado, como ocurre al presente" ${ }^{\prime 30}$.

\section{TECNOLOGÍAS ASOCIADAS}

En la carta de Wickmann a Marcuse también se puede observar cómo los instrumentos astronómicos requerían de tecnologías que los protegieran, sostuvieran, almacenaran, etc. Es decir: tecnologías asociadas al instrumento que permitieran su correcto funcionamiento, como los pivotes del círculo meridiano que afectaban su inclinación. A este respecto también existieron disputas de autoridad, sobre quienes debían hacerlo y cómo debían diseñarse, instalarse y ajustarse. Tal es el caso del busca-cometas del observatorio. De acuerdo con Wickmann, el busca-cometas había sido armado a principios de 1886 y tras el examen del mecánico Grosch, se mandó a construir en la fundición Klein "la base de fierro necesaria para la altura polar"31. Realizado esto, el instrumento fue trasladado a petición de Vergara a un edificio construido con ese propósito por el arquitecto Eloi Cortinez, pero que se erigió "según las ideas del señor Marcuse"32. Las piezas que faltaban para instalar completamente el instrumento fueron trabajadas en la fundición Klein Hermanos.

Al respecto de este instrumento, la controversia entre los trabajadores del OAN permite observar las complejidades de la apropiación cuando los instrumentos están construidos para ser usados de una manera determinada o, en este caso, en una localidad diferente a la de creación. En la discusión sobre el busca-cometas, por ejemplo, Grosch manifestaba la limitación de este instrumento en su contexto de uso en Chile, el cual tuvo que ser intervenido agregándole una pieza "para afianzar el eje del círculo horario en su movimiento giratorio. Ella fue mandada construir, según indicaciones de Grosch, en el establecimiento de los señores Klein" ${ }^{\prime 3}$.

Otro es el caso del montaje y uso del gran círculo meridiano de 6 pulgadas que requería pilastras, cuyo diseño había estado a cargo de Marcuse. Para montar el instrumento, Marcuse mandó a construir en el ala izquierda del observatorio dos pilastras de ladrillo y cemento. De acuerdo con el mecánico "la construcción fue mal dirigida, quedando las pilastras muy abiertas, y, a mi juicio, inapropiada para su destino, que [era] examinar por medio de ellos el instrumento con toda escrupulosidad"34. Según Grosch habría sido mejor montar el instrumento sobre unos caballetes de madera $y$, luego, haber generado un plano para construir las pilastras.

Cuando esto fue denunciado ante el congreso, el ministro de Instrucción Pública pidió un informe al arquitecto Cortinez, quien explicó al ministro que realizó los trabajos en el observatorio siguiendo las instrucciones de Marcuse, quien no le había proporcionado ningún plano para realizar los trabajos. A juicio del arquitecto, el problema fue que el ladrillo absorbía humedad y la transmitía hacia arriba, advertencia que fue ignorada por el astrónomo, argumentando que un arquitecto "no podía tener más conocimientos que él en estos asuntos"35. El conflicto entre los astrónomos y el mecánico o el arquitecto revelaba la disputa por la autoridad para decidir cuándo un instrumento estaba funcionando.

¿Cómo se definió la controversia? Finalmente, la resolución en torno al estado de los instrumentos y su responsabilidad en la deficiente producción científica de la institución estuvo en manos del poder político, representado por los diputados y ministros de Estado. De esta forma, en septiembre de 1886, el director del observatorio y ministro del Interior, José Ignacio Vergara, solicitó al ministro de Instrucción Pública la cancelación del contrato del primer astrónomo Adolfo Marcuse. Las razones del director para esta petición fueron los escasos trabajos que éste había realizado en los siete meses que había servido al OAN, así como su insuficiente valor científico. El astrónomo fue desvinculado de la institución ese mismo año. La disputa había concluido. 


\section{CONCLUSIONES}

La controversia en torno a los instrumentos del Observatorio Astronómico Nacional en la administración de Vergara ha permitido analizar cómo los observatorios estatales dependían estrechamente de la esfera política: su financiamiento y fiscalización pasaba por el Congreso Nacional, pero también las instituciones científicas eran administradas por políticos de la época. Sin embargo, no todos los parlamentarios estaban de acuerdo con esta imbricada relación. Para algunos existía una imposibilidad radical de realizar ambas tareas (las científicas y las políticas) de manera eficiente. La situación del OAN se volvió el ejemplo concreto de las consecuencias que esta doble tarea podía generar.

Un aspecto que resultaba esencial para la evaluación positiva del quehacer científico en la esfera política era el correcto funcionamiento de los instrumentos de la institución. Este buen funcionamiento, le permitía (a juicio de los diputados) el cumplimiento de las tareas públicas y, por ende, justificaba el financiamiento estatal del quehacer científico. En la evaluación de este quehacer estaban en disputa no solo los juicios, sino la autoridad de quienes los emitían ¿quién podía juzgar mejor la calidad de los instrumentos y del conocimiento científico que posibilitaban: el político, el astrónomo, el mecánico o el arquitecto? Como se ha podido ver en diferentes relatos de los involucrados, esta es una permanente disputa

\section{NOTAS}

1 Trabajo financiado por proyecto FONDECYT Regular № 1170625. Los autores agradecen al programa su apoyo.

2 Para una tipología de las controversias: (Sismondo, Sergio 2010, pp. 120-135), en especial el capítulo "Controverses".

3 En los estudios de las trayectorias tecnológicas este problema ha sido abordado profusamente. Ver al respect: Dosi, Giovanni, (1982); Cohen, Wesley M., y Levinthal, Daniel A. (1990); Viotti, Eduardo, (2002).

4 Manuel Gregorio Balbontín Viancos, era abogado, titulado de la Facultad de Derecho de la Universidad de Chile en 1870. Se dedicó a su profesión y al ejercicio del periodismo. En 1884 fue nombrado Director General en la II Convención Nacional del Partido Conservador. En 1885 fue electo Diputado por Castro. Al respecto ver: Figueroa, Virgilio (1928); Bolados Carter, Alfredo (1918).

5 Sesiones Extraordinarias de la Cámara de Diputados en 1885-1886, sesión del 3 de febrero de 1886, Santiago, Imprenta Nacional, p. 627. entre quienes reparan instrumentos, construyen tecnologías asociadas y quienes los usan.

En este caso, epistémicamente, no se resuelve la autoridad en esta materia, pero sí hay una resolución práctica que zanja la discusión pública: finalmente quien determina el problema y los culpables es el político. Pese a esto, el debate acerca del uso y mantenimiento de los instrumentos permite observar el papel que jugaron los técnicos, ingenieros y constructores. Estos, al ejercer las acciones de reparación y mantención sobre los instrumentos, tuvieron en sus manos la posibilidad de habilitar las prácticas cotidianas dentro del observatorio: reparar un busca-cometas o círculo mediano era lo que posibilitaba cumplir con las labores que se asignaban a la institución. No obstante, cuando la reparación era deficiente o cuestionada entre los mismos miembros del OAN, se tensionaba y desarticulaba el orden institucional, entorpeciendo el trabajo científico y dando pie para las críticas desde el mundo político.

En una línea similar, la necesaria adaptación de los instrumentos a condiciones diferentes a las existentes en los países de fabricación, o la urgencia de crear tecnologías asociadas, requirieron de soluciones locales. Allí, los actores no propiamente "científicos" se volvieron más relevantes que los astrónomos gracias a que construyeron elementos claves como soportes, resguardos y protecciones que funcionaron de forma inseparable de los instrumentos, permitiendo su práctica científica diaria.
6 Domingo Santa María, abogado titulado de la Universidad de Chile en 1847. Trabajó de profesor de geografía, aritmética e historia en el Instituto Nacional en 1845 y como profesor en la Facultad de Filosofía de la Universidad de Chile, además de asumir diversos cargos políticos en diferentes periodos, como diputado, senador, intendente y ministro. Fue electo presidente de la República en 1881, representando al Partido Liberal. Al respecto ver: Figueroa, Pedro (1901); Figueroa, Virgilio (1931).

7 José Ignacio Vergara Urzúa, ingresó a estudiar en la Facultad de Ingeniería de la Universidad de Chile en 1856 y obtuvo el título de Ingeniero Geógrafo en 1863. Desde 1860 se desempeñó como ayudante del Observatorio Astronómico Nacional. Fue nombrado Director interino en 1865 y en propiedad a partir de 1874 . Presidente de la Sociedad de Instrucción Primaria entre 1870 - 1874, ayudó a fundar la Escuela Italia. Fue también profesor de Mecánica, Astronomía y Cálculo diferencial e integral en la Universidad de Chile y uno de los fundadores de la Oficina Central de Meteorología con Ignacio Domeyko en 1868. 
Por sus observaciones meteorológicas, obtuvo en 1875 una medalla en el Congreso Geográfico de Paris. Al respecto ver: Figueroa, Pedro (1901), pp. 433-434; Figueroa, Virgilio (1931), p. 1029.

8 Mientras Vergara fue director del OAN, ejerció paralelamente diversos cargos públicos, dado que no existía en el país ninguna normativa que se lo impidiera y hasta ese momento no había recibido cuestionamientos por ello.

9 Marcuse alcanzó a trabajar en la institución por tan sólo nueve meses.

10 Sesiones Extraordinarias de la Cámara de Diputados en 1885-1886, sesión del 3 de febrero de 1886, Santiago, Imprenta Nacional, p. 627.

11 Guillermo PuelmaTupper estudió medicina en la Universidad de Chile y en 1872 ayudó frente a una epidemia de viruela en Santiago, siendo condecorado por su trabajo en este tema. En 1873 se hizo miembro de la Academia de Bellas Letras y publicó varios textos de poesía. En 1876 viajó a Europa con el propósito de seguir los estudios médicos en la Universidad de Heidelberg y Universidad de Magburg, obteniendo superfeccionamiento en Fisiología. En 1878 fue director de la Escuela Abraham Lincoln y en 1879 ingresó al servició médico y ayudó a fundar el Asilo de Inválidos de la Guerra del Pacífico, participando en la Sociedad Médica de Santiago y siendo nombrado profesor extraordinario de Histología en la Universidad de Chile. En 1881 se comenzó a dedicar al periodismo político, redactando y dirigiendo el periódico "La Época" hasta 1884, destacándose por ser un reconocido defensor del positivismo de Comte. También fue colaborador en el periódico "La libertad Electoral". Miembro del Partido Radical, fue diputado por Parral entre 1882 y 1885 y diputado por San Carlos entre 1885 y 1888 . Fue opositor del gobierno de Domingo de Santa María y se destacó por su férrea oposición al presupuesto de Culto y sus posturas antirreligiosas. Al respecto ver: Figueroa, Virgilio (1931), p. 571; Figueroa, Pedro Pablo (1897).

12 Sesiones Extraordinarias de la Cámara de Diputados en 1885-1886, sesión del 3 de febrero de 1886, Santiago, Imprenta Nacional, p. 627.

13 Adolf Marcuse fue un astrónomo alemán. Estudió en la Universidad de Estrasburgo y Berlín, obteniendo su doctorado en 1884. En 1882, fe asistente en la Expedición Alemana para observar el tránsito de Venus en los Estados Unidos. Llegó a trabajar al OAN en febrero 1886, desempeñando el cargo de primer astrónomo. Su contrato fue cancelado a fines de ese mismo año. Tras su partida de Chile, trabajó en el Observatorio Astronómico de Berlín entre 1888 y 1891.

14 Sesiones Extraordinarias de la Cámara de Diputados en 1885-1886, sesión del 24 de julio de 1886, Santiago, Imprenta Nacional, p. 337.

15 Sesiones Extraordinarias de la Cámara de Diputados en 1885-1886, sesión del 12 de agosto de 1886, Santiago, Imprenta Nacional, pp. 637 - 638.

16 Guillermo Wickmann, "Rectificación al señor Puelma Tupper", El Ferrocarril, 31 de agosto de 1886, p.2. De acá en adelante se ha conservado la grafía original de los documentos.

17 Jacinto Chacón Barrios se tituló de abogado en 1843. Ejerció también como poeta, profesor y periodista, colaborando en 1842 en el periódico "El Semanario de Santiago" y en 1844 en "El Crepúsculo". Desde 1851 fue redactor de "El Mercurio de Valparaíso" y durante la primera mitad de la década de 1860 fue director de la "Revista del Pacífico". Pese a ser profundamente religioso, militó en el Partido Liberal, siendo diputado suplente por San Felipe entre 1885 a 1888. Al respecto ver: Figueroa, Virgilio (1928), Diccionario histórico, biográfico y bibliográfico de Chile. Tomo II, Santiago de Chile, Balcells \& Co., p. 517-518; Figueroa, Pedro Pablo (1897), pp. 320-322.

18 Chacón, Jacinto (1886), p. 4.

19 Anónimo, "La Quinta Normal. Sus establecimientos agronómicos i científicos", La Época, 17 de julio de 1886, p.3.

20 Incluso por dichos trabajos obtuvo en 1875 una medalla en el Segundo Congreso Geográfico de París.

21 Chacón, Jacinto (1886), p. 99.

22 Chacón, Jacinto (1886), p. 99. Estas consideraciones están en concordancia con los requerimientos de la propia disciplina a fines del siglo XIX. En efecto, un aspecto fundamental en el estudio de los astros fue la expansión de las técnicas de observación. En este punto la calibración de los instrumentos y la homogeneización de los procesos de tratamiento de datos (Licoppe en Bourguet et al., 2002) y, por otro lado, las estrategias sociales subyacentes a estas prácticas, tales como los métodos de administración, las formas de organización del trabajo, etc. (Aubin et al., 2010; Raposo 2014) fueron fundamentales. Desde este punto de vista, la repetición de técnicas y procedimientos de observación a lo largo de los observatorios del mundo, no sólo implicó la formación de una comunidad científica, como su profesionalización (Lankford, 1997), sino también el control y organización de las redes de observación que las sustentaban (Rothenberg, 1981; Lankford, 1981). Lo anterior, al mismo tiempo, permitió diferenciar entre una disciplina legitimada desde tales protocolos y procedimientos, de una astronomía más bien hecha por amateurs (Ogilvie, 2000). Estos requerimientos globales también se aplicaron a los observatorios de América del Sur, como Argentina (Minniti y Paolantonio, 2009; Rieznik, 2010, 2011, 2013 y Brasil (Barboza, 2010).

23 Sesiones Extraordinarias de la Cámara de Diputados en 1885-1886, sesión del 12 de agosto de 1886, Santiago, Imprenta Nacional, p. 447. Estos datos fueron recibidos por la Cámara de Diputados el 17 de agosto. El Ferrocarril, Santiago de Chile, 18 de agosto de 1886, p. 1.

24 Archivo Nacional Histórico de Chile (ANHCh), Fondo Ministerio de Educación (FME), vol. 554, "Representación del señor Marcuse dirijida al Supremo Gobierno", sección II, $\sin$ fecha.

25 Luis Grosch, alemán contratado en 1852 por el primer director del OAN (Carlos Moesta) para que sirviera como ingeniero óptico. Se desempeñó en esta función dentro de la institución hasta que se jubiló en 1892, ocupándose también de las observaciones meteorológicas. Falleció en 
1902. Greve, Ernesto (1944), p.133; Friedrich, Wilhelm Ristenpart (1910), pp.12-13.

26 ANHCh, FME, vol. 554, Documento №8, Carta de Wickmann a Cruls, 20 de septiembre de 1885 .

27 ANHCh, FME, vol. 554, Documento №9, Carta de Wickmann a Marcuse, 25 de diciembre de 1885 .

28 ANHCh, FME, vol. 554, Documento №9, Carta de Wickmann a Marcuse, 25 de diciembre de 1885 .

29 ANHCh, FME, vol. 554, Documento №9, Carta de Wickmann a Marcuse, 25 de diciembre de 1885

30 "Documentos relativos a los trabajos de este establecimiento remitidos por el señor Ministro de Justicia a la Honorable Cámara de Diputados y mandados a publicar el 17 del corriente" (1886), Santiago, Imprenta Nacional, p.18. Cursivas del texto original. En esta disputa de saberes también se evidencia lo que algunos autores como Edgerton y Henke han propuesto respecto a que quienes realizan tareas de reparación muchas veces se sitúan en una posición que les permite cuestionar los usos de los objetos e, incluso, a los diseñadores de los mismos. Los reparadores generan nuevos saberes en torno a las prácticas y relaciones con los objetos que interactúan (Edgerton, 2008; Henke, 1999).

31 "Documentos relativos a los trabajos de este establecimiento remitidos por el señor Ministro de Justicia a la Honorable Cámara de Diputados y mandados a publicar el
17 del corriente" (1886), Santiago, Imprenta Nacional, p.15.

32 "Documentos relativos a los trabajos de este establecimiento remitidos por el señor Ministro de Justicia a la Honorable Cámara de Diputados y mandados a publicar el 17 del corriente" (1886), Santiago, Imprenta Nacional, p.15.

33 El detalle de las piezas se encuentra en un anexo presentado por Klein. Estas piezas se mandaron a construir en 1885 y son: pieza f. acepillada número 314, a 35 centavos \$109; gastos de modelo, \$25; Recortado 2 esquinas en la parte quebrada de la pieza vieja i ajustado todo en la pieza nueva, \$25. "Documentos relativos a los trabajos de este establecimiento remitidos por el señor Ministro de Justicia a la Honorable Cámara de Diputados y mandados a publicar el 17 del corriente" (1886), Santiago, Imprenta Nacional, p. 21.

34 Documentos relativos a los trabajos de este establecimiento remitidos por el señor Ministro de Justicia a la Honorable Cámara de Diputados y mandados a publicar el 17 del corriente" (1886), Santiago, Imprenta Nacional, p.17.

35 "Documentos relativos a los trabajos de este establecimiento remitidos por el señor Ministro de Justicia a la Honorable Cámara de Diputados y mandados a publicar el 17 del corriente" (1886), Santiago, Imprenta Nacional, p.19.

\section{BIBLIOGRAFÍA}

Barboza, C.H. da Motta (2010), "Ciência e natureza nas expedições astronômicas para o Brasil (1850-1920)", Bol. Mus. Para. Emílio Goeldi. Cienc. Hum., Belém 5, (2), pp. 273-294.

Beder, Sharon (1991), "Controversy and Closure: Sydney's Beaches in Crisis", Social Studies of Science, 21 (2), pp. 223-256.

Bijker, Wiebe; Hughes, Thomas y Pinch, Trevor (1987), The Social Construction of Technological Systems: New Directions in the Sociology and History of Technology, Cambridge/ Londres: MIT Press.

Bolados, Alfredo (1918), Album del Congreso Nacional en su primer centenario 1818 - 1918, Santiago de Chile, Imprenta España.

Bourguet, MN; Licoppe, C. y H.O. Sibum (2002), Instruments, Travel and Science. Itineraries of precision from the seventeenth to the twentieth century, London Routledge.

Cambrosio, Alberto; Keating, Peter y MacKenzie, Donald (1990), "Scientific practice in the courtroom: The construction of sociotechnical identities in a biotechnology patent dispute", Social Problems, 37 (3), pp. 275-293.
Chacón, Jacinto (1886), La Quinta Normal y sus establecimientos agronómicos y científicos. Paseo de estudio, Santiago de Chile, Imprenta Nacional.

Cohen, Wesley; Levinthal, Daniel (1990), "Absorptive capacity: A new perspective on learning and innovation", Administrative Science Quarterly, 35 (1), pp. 128 -152.

Dascal, Marcelo (1998), "The Study of Controversies and the Theory and History of Science", Science in Context, 11 (2), pp. 147-154.

Dosi, Giovanni (1982), "Technological paradigms and technological trajectories: A suggested interpretation of the determinants and directions of technical change", Research Policy, 11(3), pp. 147-162.

Edgerton, David (2008), The shock of the old. Technology and global history since 1900, Londres, Profile Books.

Edwards, Paul; Hecht, Gabrielle (2010), "History and the Technopolitics of Identity: The Case of Apartheid South Africa", Journal of Southern African Studies, 36 (3), pp. 619-639.

Engelhardt, Tristram; Caplan, Arthur (eds.) (1987), Scientific Controversies Case Studies in the Resolution and Closure of Disputes in Science and Technology, Cambridge, Cambridge University Press. 
Figueroa, Virgilio (1928), Diccionario histórico, biográfico y bibliográfico de Chile. Tomo II, Santiago de Chile, Balcells \& Co.

Figueroa, Virgilio (1931), Diccionario histórico, biográfico y bibliográfico de Chile. Tomos IV-V, Santiago de Chile, Imprenta La Ilustración.

Figueroa, Pedro Pablo (1897), Diccionario biográfico de Chile. Tomo II, Santiago de Chile, Imprenta Barcelona.

Figueroa, Pedro Pablo (1901), Diccionario biográfico de Chile. Tomo III, Santiago de Chile, Imprenta Barcelona.

Freudenthal, Gideon (1998), "Controversy", Science in Context, 11 (2), pp. 155-160.

Friedrich, Wilhelm Ristenpart (1910), Astrónomos Alemanes en Chile, Santiago de Chile, Imprenta Universitaria, pp. 12-13.

Graham, Stephen; Nigel Thrift (2007), “Out of Order. Understanding Repair and Maintenance", Theory, Culture \& Society, 24(3), pp. 1-25.

Greve, Ernesto (1944), Historia de la Ingenieria en Chile, tomo III, Santiago de Chile, Editorial Universitaria, p. 133.

Henke, Christopher (1999), "The Mechanics of Workplace Order: Toward a Sociology of Repair", Berkeley Journal of Sociology, 44, pp. 55-81.

Jackson, Steven (2014), "Rethinking Repair". En: Gillespie, Tarleton; Boczkowski, Pablo y Foot, Kirsten (eds.), Media Technologies: Essays on Communication, Materiality, and Society, Cambridge, MIT Press, pp.221-240.

Jasanoff, Sheila (1996), Science at the bar: law, science and technology in America, Cambridge, Harvard University Press.

Lankford, J. (1981), “Amateurs versus Professionals: The Controversy over Telescope Size in Late Victorian Science", Isis, 72, pp. 11-28.

Lankford, J. (1997), American Astronomy: Community, Careers, and Power, 1859-1940, Chicago, University of Chicago Press.

Latour, Bruno (1991), “Pasteur y Pouchet. Heterogénesis de la historia de las ciencias". En: Serres, Michel (coord.), Historia de las ciencias, Madrid, Cátedra, pp. 477-502.

Livingstone, David (2007), "Science, site and speech scientific knowledge and the spaces of rhetoric", History of the Human Sciences, 20 (2), pp. 71-98.

Machamer, Peter; Darden, Lindley y Craver, Carl (2000), "Thinking about Mechanisms", Philosophy of Science, 67 (1), pp. $1-25$.

MacKenzie, Donald (1978), "Statistical Theory and Social Interest: A case study", Social Studies of Science, 8 (1), pp. 35-83.
Minnitti, E.; Paolantonio, S. (2009), Córdoba estelar: desde los sueños a la astrofísica: historia del Observatorio Nacional Argentino, Córdoba, Argentina, Universidad Nacional de Córdoba.

Morus, Iwhan (2016), "Invisible technician, Instruments-makers and Artisans". En: Lightman, Bernard (ed.), A companion to thehistory of science, Willey-Blackwell, pp. 95-110.

Nieto-Galan, Agustí (2011), Los Públicos de la Ciencia. Expertos y profanos a través de la historia, Madrid, Marcial Pons.

Ogilvie, M.B. (2000), “Obligatory amateurs: Annie Maunder (1868-1947) and British women astronomers at the dawn of professional astronomy", The British Journal for the History of Science, 33 (1), pp. 67-84.

Portales, Felipe (2004), Los mitos de la democracia chilena. Tomo I. Desde la Conquista hasta 1925, $1^{\circ}$ edición, Santiago de Chile, Catalonia,

Potthast, Jörg (2007), Die Bodenhaftung der Netzwerkgesellschaft, Bielefeld, Transcript.

Raposo, Pedro (2014), "Time, Weather and Empires: The Campos Rodrigues Observatory in Lourenço Marques, Mozambique (1905-1930)", Annals of Science, 72 (3), pp. 279-305.

Rieznik, M. (2010), “El Bureau des Longitudes y la fundación del Observatorio de La Plata en la Argentina (1882-1890)", História, Ciências, Saúde - Manguinhos, 17 (3), pp. 679-703.

Rieznik, M. (2011), Los cielos del sur. Los observatorios de Córdoba y de La Plata, 1871-1920, Rosario, Prohistoria.

Rieznik, M. (2013), "The Cordoba Observatory and the history of the personal equation' (1871-1886)", Journal for the History of Astronomy, Cambridge, 44 (156), pp. 277-301.

Schaffer, Simon (2011), "Easily Cracked. Scientific Instruments in States of Disrepair", Isis, 102 (4), pp.706-717, p.705.

Shapin, Steven (1989), "The invisible technician", American Scientist, 77 (6), pp. 554-563.

Shapin, Steven; Barnes, Barry (1977), "Science, nature and control: Interpreting mechanics institutes", Social Studies of Science, 7(1), pp. 31-74.

Shapin, Steven; Schaffer, Simon (1985), Leviathan and the AirPump: Hobbes, Boyle, and the Experimental Life. Princeton, Princeton University Press.

Sismondo, Sergio (2010), An Introduction to Science and Technology Studies, Wiley-Blackwell.

Viotti, Eduardo (2002), “National learning systems: A new approach on technical change in late industrializing economies and evidences from the cases of Brazil and South Korea", Technological Forecasting and Social Change, 69 (7), pp.653-680. 
